\title{
A new structural health monitoring system for real-time evaluation of building damage
}

\author{
Koichi Kusunoki ${ }^{1 *}$ \\ ${ }^{1}$ Earthquake Research Institute, the University of Tokyo, 1-1-1, Yayoi, Bunkyo Ku, Tokyo, Japan
}

\begin{abstract}
The author has developed a new method for evaluating the seismic performance of existing structures from measured accelerations based on the capacity spectrum method. This involves comparing the performance curve, which is the equivalent nonlinear behavior of a simplified single-degree-of-freedom system, and the demand curve, which is the relationship between the response acceleration and displacement spectra. Two telecommunication towers in Japan were instrumented in 2016, and their responses during several earthquakes have been recorded. This paper discusses the evaluation of damage during the two earthquakes. Moreover, parameters such as the predominant period and the required performance are discussed. The proposed system evaluated both towers as being "elastic." The damping ratios of the towers are very low, which caused the oscillations to continue for more than 5 min after the mainshock of each earthquake because of long-period components of the seismic motion.
\end{abstract}

\section{Introduction}

In 2000, a new structural calculation method known as the "response and limit-capacity calculation" was added to the Building Standard Law of Japan as a new option for evaluating the seismic performance of buildings. The method involves comparing the performance and demand curves of a building. The performance curve represents the building's nonlinear behavior as a simplified singledegree-of-freedom (SDoF) system, whereas the demand curve represents the force-deformation design criteria defined in the building code.

Meanwhile, when a strong earthquake occurs, a quick inspection of the damage to buildings in the affected area is very important for reducing the damage caused by aftershocks. However, the current inspection method this involves structural engineers making visual assessments, which is both time consuming and subjective. To overcome these drawbacks, a new real-time method has been proposed [1-4] for evaluating building damage with inexpensive accelerometers based on the aforementioned response and limit-capacity calculation.

In 2014, two steel towers used for microwave telecommunication in Tokyo and Yokohama in Japan were instrumented and had been monitored since then. One tower was instrumented because it was seen to continue oscillating after the ground had ceased to shake during the 2011 Tohoku Earthquake. Since the towers were instrumented, two major earthquakes have occurred in the region. One was the Fukushima Offshore Earthquake of magnitude 7.4 at 0559 JST on November 22, 2016. At both tower sites, the maximum seismic intensity was recorded as three on the Japan Meteorological Agency (JMA) seismic intensity scale.
The other earthquake was the northern part of Ibaraki Prefecture Earthquake of magnitude 6.3 at 2138 JST on December 28, 2016. Again, the maximum seismic intensity at each tower site was 3 . By using the proposed system, both towers were evaluated as being elastic during those 2016 earthquakes. This paper discusses the damage evaluation results and design parameters such as the predominant period and the required performance.

\section{Outline of towers and measurements}

Hazawa Tower [Fig. 1(a)] is a cylindrical steel tower in Yokohama, Japan, that is $58 \mathrm{~m}$ tall and is instrumented with accelerometers [ITK-002, shown in Fig. 2(a)] at its base and top and $1 / 3$ and $2 / 3$ of its total height, as indicated in Fig. 1(a). The accelerometers are sampled at a rate of $100 \mathrm{~Hz}$ with a 24-bit A/D converter. The accelerometers are three-dimensional ones with a maximum measurable acceleration of $2,450 \mathrm{~cm} / \mathrm{s} 2$ and a noise level of no more than $0.1 \mathrm{gal} / \mathrm{s}$. The acceleration data are stored for $24 \mathrm{~h}$ on a server that is located in the tower, and that is connected to the outside world through the cellphone network. The data can be accessed through the Internet.

Higashi Oshima Tower [Fig. 1(b)] is another cylindrical steel tower and is located in Tokyo, Japan. It is $63 \mathrm{~m}$ tall and is instrumented with accelerometers [IoLAM-01, shown in Fig. 2(b)] at its base and top and half its total height. The accelerometers are sampled at a rate of $100 \mathrm{~Hz}$ with a 24-bit A/D converter. The accelerometers are three-dimensional ones with a maximum measurable acceleration of $3,430 \mathrm{~cm} / \mathrm{s} 2$ and a noise level of no more than $0.1 \mathrm{Gal} / \mathrm{s}$. As with Hazawa

\footnotetext{
* corresponding author: kusunoki@eri.u-tokyo.ac.jp
} 
Tower, the acceleration data are stored for $24 \mathrm{~h}$ on a server that is located in the tower, the server is connected to the outside world through the cell-phone network, and the data can be accessed through the Internet.

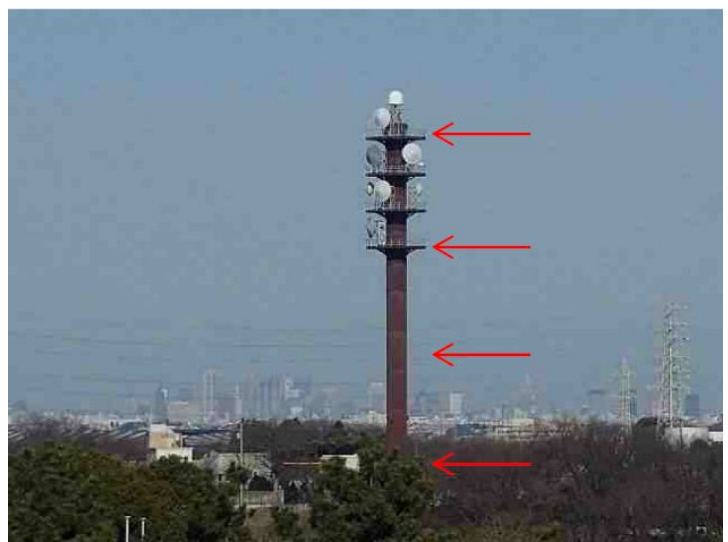

(a) Hazawa Tower

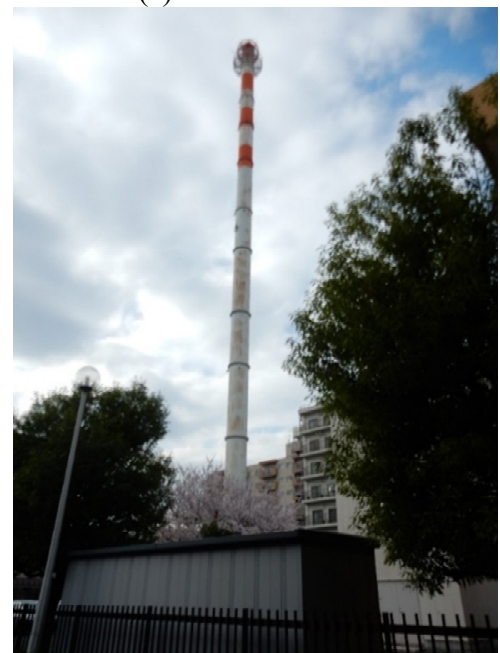

(b) Higashi Oshima Tower

Fig. 1 Target towers

\section{Measured accelerations}

\subsection{Fukushima Ken Oki Earthquake (November 22, 2016)}

At 0559 JST on November 22, 2016, a strong earthquake of magnitude 7.4 occurred $30 \mathrm{~km}$ beneath the seafloor off the shore of Fukushima Prefecture in Japan. The earthquake epicenter and the tower locations are shown in Fig. 3. A JMA seismic intensity of 3 was measured at each site, and the responses of the towers during the earthquake were stored successfully.

Fig. 4 shows the calculated transfer functions of the NS direction from the top, $2 / 3$, and $1 / 3$ heights to the bottom of Hazawa Tower. The predominant frequency was $0.6897 \mathrm{~Hz}$, which corresponds to a period of $1.45 \mathrm{~s}$. The amplification factor associated with the top of the tower was roughly 150 , which means that the damping coefficient of the tower is very low.

Fig. 5 shows the acceleration time histories of the predominant NS direction measured at the bottom and top of Hazawa Tower. As shown in Fig. 5(a), the ground acceleration reached its peak at roughly $80 \mathrm{~s}$, but a small

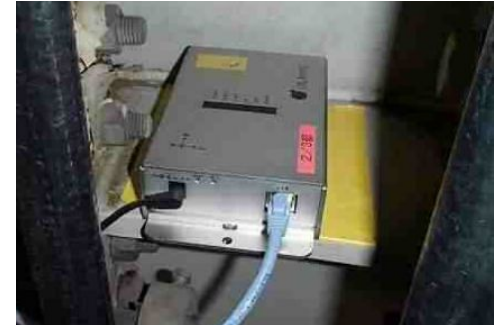

(a) ITK-002 for Hazawa Tower

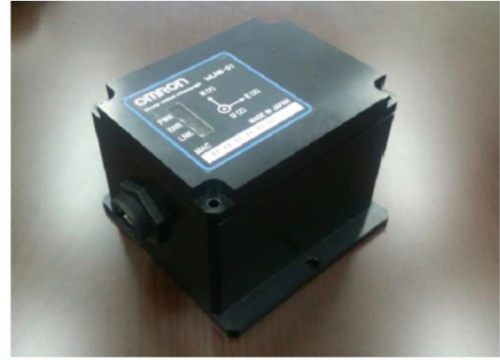

(b) IoLAM for Higashi Oshima tower

Fig. 2 Instrumented sensors

long-period component can be observed after $150 \mathrm{~s}$. Because of this long-period component, the acceleration at the top reached its peak at roughly $170 \mathrm{~s}$, at which time the acceleration at the bottom was very small. The longperiod component may be why the tower was seen to continue oscillating after the ground had ceased to shake during the 2011 Tohoku Earthquake.

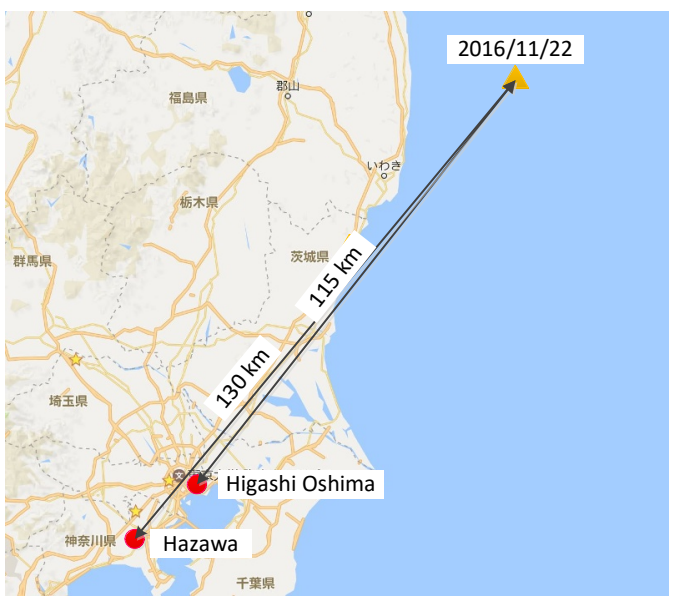

Fig. 3 Earthquake epicenter (triangle) and tower sites (circles)

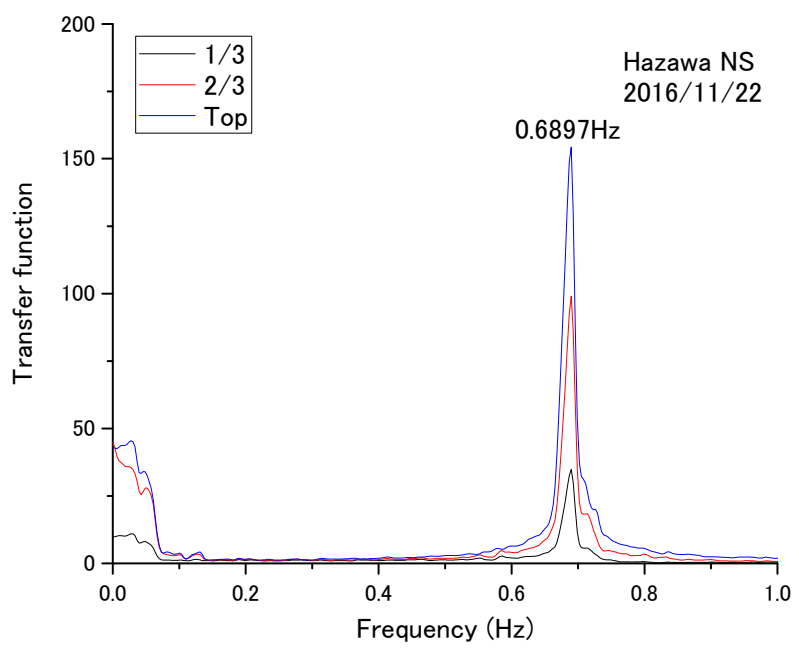

Fig. 4 Transfer functions (NS direction) 


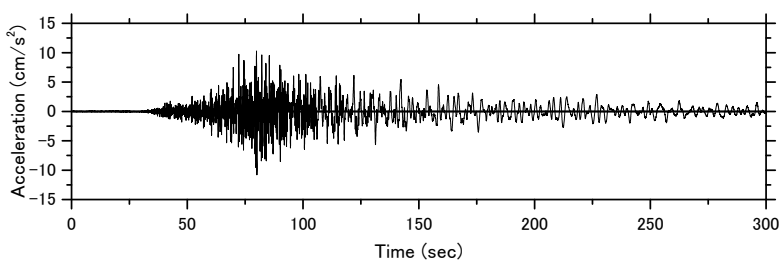

(a) Basement

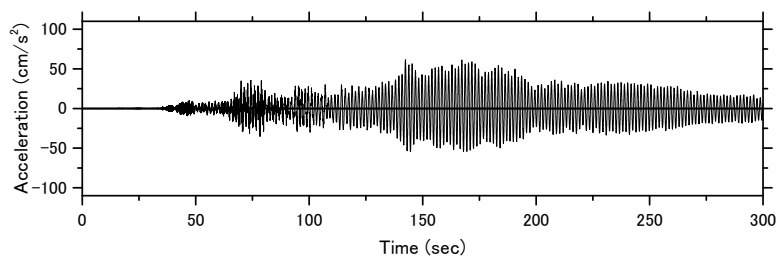

(b) Top

Fig. 5 Measured accelerations of Hazawa Tower (NS direction)

\subsection{Ibaraki Ken North Earthquake (December 28,} 2016)

At 2138 JST on December 28, 2016, a strong earthquake of magnitude 6.3 occurred $10 \mathrm{~km}$ beneath the ground surface in the north of Ibaraki Prefecture in Japan. The earthquake epicenter and the tower locations are shown in Fig. 6. A JMA seismic intensity of 3 was measured at each site, and the responses of the towers during the earthquake were stored successfully.

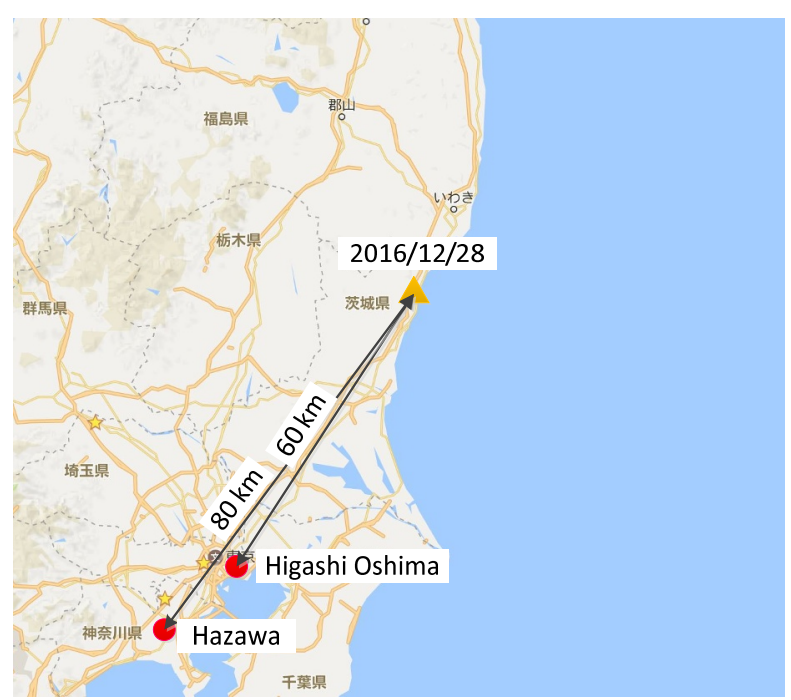

Fig. 6 Earthquake epicenter (triangle) and tower sites (circles)

Fig. 7 shows the calculated transfer functions of the NS direction from the top and half-way up Higashi Oshima Tower to the bottom. The predominant frequency was $0.415 \mathrm{~Hz}$, which corresponds to a period of $2.41 \mathrm{~s}$. The amplification factor associated with the top was roughly 175 , which means that the damping coefficient of this tower is also very low.

Fig. 8 shows the acceleration time histories of the predominant NS direction measured at the bottom and top of Higashi Oshima Tower. As shown in Fig. 8(a), the ground acceleration reached its peak at roughly $30 \mathrm{~s}$, but a very small long-period component can be observed until roughly $200 \mathrm{~s}$. Because of this long-period component and the relatively light damping, the top reached its peak acceleration at roughly $55 \mathrm{~s}$ and was still shaking at 300 $\mathrm{s}$, by which time the acceleration at the bottom was effectively zero.

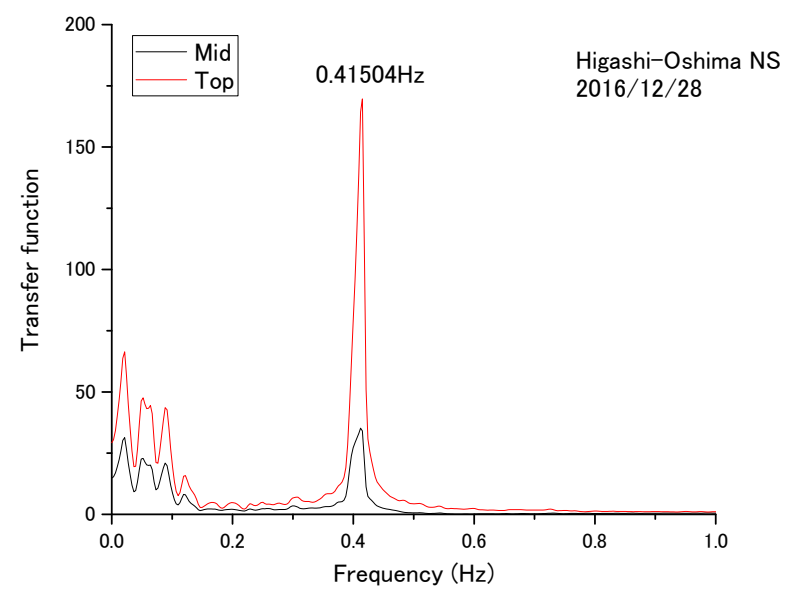

Fig. 7 Transfer functions (NS direction)

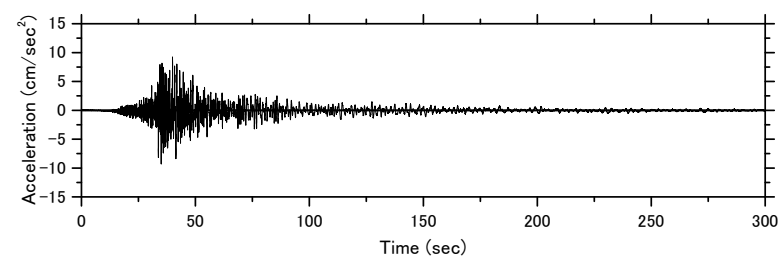

(a) Basement

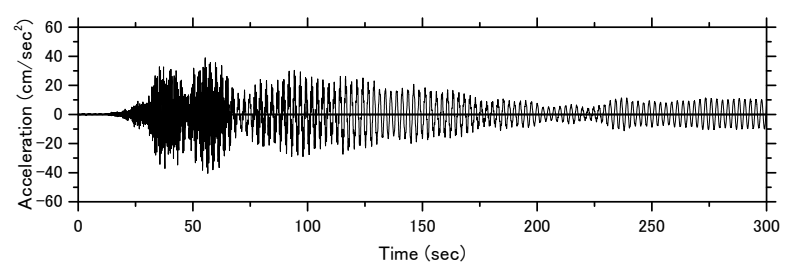

(b) Top

Fig. 8 Measured acceleration at Higashi Oshima Tower (NS Direction)

\section{Damage evaluated from measured accelerations}

The outline of the evaluation is shown in Fig. 9. The maximum responses during a main shock and aftershock are estimated as the intersection of the capacity and demand curves. The capacity curve is the relationship between the representative restoring force and representative displacement, which are derived from the measured accelerations. The demand curve is the relationship between the response acceleration spectrum and response displacement spectrum, which are derived from the acceleration at the basement of the building. The amount of the damping coefficient needs to be assumed when the demand curve is derived. The damping coefficient for the elastic stage can be assumed as the viscous damping ratio of $5 \%$ as "Curve 1 " shown in Fig. 1. When the building experience yielding as point $(\mathrm{A})$ in Fig. 9, an additional damping effect due to non-linear response needs to be considered. Since the additional damping effect increases corresponding to the damage of 
the building, the total damping coefficient increases according to the representative displacement. Therefore, the demand curve is reduced from point (B) as "Curve 2" in Fig. 9. The maximum response during the main shock is predicted as the intersection of the capacity curve and the reduced demand curve (Curve 2), point (C) in Fig. 9.

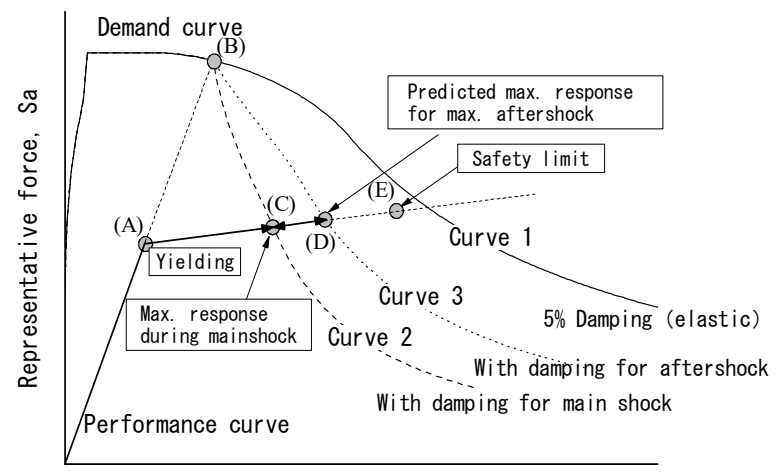

Representative displacement, Sd

Fig. 9 Performance curve and demand curve

On the other hand, the same method can be applied to predict the maximum response during an aftershock with considering the main shock and the following aftershock as one very long duration earthquake. The input energy of the combined earthquake is consequently larger than that of the main shock; then the maximum response may be larger than that of the main shock. It means that the equivalent damping effect becomes smaller than that of only the main shock as "Curve 3" shown in Fig. 9. The predicted maximum response during the aftershock is the intersection of Curve 3 and the capacity curve, with the assumption that the maximum aftershock is the same as the main shock.

In order to evaluate the safety of the building, the first mode of the response needs to be taken out to derive the capacity curve. The ultimate point is defined with the safety limit of each story. The maximum story drift of each story is derived from the maximum representative displacement and the first mode shape. Since the proposed safety evaluation is based on the first mode, the higher mode effect needs to be considered separately, if the higher mode effect is negligible such as high-rise buildings.

\section{Simplification method of MDoF system to SDoF system ${ }^{7), 8), ~ 9), ~ 10), ~ 14) ~}$}

In this section, the simplification method to SDoF system, which is used in practice as the equivalent linearization method, is introduced. In practice, the restoring force and response relative displacement to the basement are obtained from the static nonlinear analysis (pushover analysis). The equation of the motion for MDoF system is shown as Equation (1).

$$
[M]\{\ddot{x}\}+[C]\{\dot{x}\}+[K]\{x\}-[M]\{1\} \ddot{x}_{0}
$$

Where, $[\mathrm{M}]$ : mass matrix, $[\mathrm{C}]$ : damping matrix, $[\mathrm{K}]$ : stiffness matrix, $\{\ddot{x}\}$ : relative acceleration vector to the basement, $\{\dot{x}\}$ : relative velocity vector to the basement, $\{x\}$ :relative displacement vector to the basement, and $\ddot{x}_{0}$ : ground acceleration.

There is a number of $\mathrm{N}$ predominant periods and predominant modes for Nth-degree-of-freedom system. By solving the eigenvalue equation, an eigen period of ${ }_{s} T$, an eigen angular frequency of ${ }_{s} \omega\left(=2 \pi /{ }_{s} T\right)$, and an eigen mode vector of $\left\{{ }_{s} u\right\}$ for s-th mode are obtained. The relative displacement vector, $\{\mathrm{x}\}$ is derived as Equation (2) from the response of each mode.

$$
\{x\}=\sum_{s=1}^{N}\left\{{ }_{s} u\right\} \cdot{ }_{s} q=[U]\{q\}
$$

${ }_{s} q$ : response displacement of s-th mode.

By substituting Equation (2) to Equation (1), N equations of ${ }_{s} q$ is obtained as Euqtion (3).

$$
\begin{aligned}
& { }_{s} \ddot{q}+2{ }_{s} h \cdot{ }_{s} \omega \cdot{ }_{s} \dot{q}+{ }_{s} \omega^{2} \cdot{ }_{s} q=-{ }_{s} \beta \cdot \ddot{x}_{0} \\
& { }_{s} \beta=\frac{\left\{{ }_{s} u\right\}^{T}[M]\{1\}}{\left\{{ }_{s} u\right\}^{T}[M]\left\{{ }_{s} u\right\}}=\frac{\sum m_{i} \cdot{ }_{s} u_{i}}{\sum m_{i}{ }_{s} u_{i}{ }^{2}}
\end{aligned}
$$

${ }_{s} h$ : Damping coefficient for $s-$ th mode, and ${ }_{s} \beta$ :Stimulation factor for s-th mod.

${ }_{s} \beta \cdot\left\{{ }_{s} u\right\}$ is the stimulation vector, which is the eigen mode for $\mathrm{s}$-th mode of unit vector of $\{1\}$ as shown in Equation (4).

$$
\{1\}=\sum_{s=1}^{N} \beta \cdot\left\{{ }_{s} u\right\}
$$

The response of each mode can be calculated as the response of the same ground acceleration of $\ddot{x}_{0}$ with ${ }_{s} q_{0}$, which is defined as Equation (5).

$$
{ }_{s} q={ }_{s} \beta \cdot{ }_{s} q_{0}
$$

With Equation (5), Equations (6) and (7) are derived from Equations (2) and (3), respectively.

$$
\begin{aligned}
& \{x\}=\sum_{s=1}^{N}{ }_{s} \beta \cdot\left\{{ }_{s} u\right\} \cdot{ }_{s} q_{0} \\
& { }_{s} \ddot{q}_{0}+2{ }_{s} h \cdot{ }_{s} \omega \cdot{ }_{s} \dot{q}_{0}+{ }_{s} \omega^{2} \cdot{ }_{s} q_{0}=-\ddot{x}_{0} \\
& s=1 \sim N
\end{aligned}
$$

The base shear of s-th mode, ${ }_{s} Q_{B}$ is calculated as Equation (8) with the assumption that the external force distribution shape is proportional to the mode shape.

$$
{ }_{s} Q_{B}={ }_{s} \bar{M} \cdot\left({ }_{s} \ddot{\Delta}+\ddot{x}_{0}\right)=\sum_{i=1}^{N} m_{i} \cdot{ }_{s} \beta \cdot{ }_{s} u_{i}\left({ }_{s} \ddot{\Delta}+\ddot{x}_{0}\right)
$$

${ }_{s} \ddot{\Delta}$ : Relative representative acceleration for s-th mode.

The equivalent mass for s-th mode, ${ }_{s} \bar{M}$, is calculated as Equation (9). 


$$
{ }_{s} \bar{M}=\frac{\left(\sum m_{i} \cdot s u_{i}\right)^{2}}{\sum m_{i} \cdot s^{2} u_{i}^{2}}
$$

The relative displacement to the basement for s-th mode is calculated as Equation (10) with the stimulation vector and the representative displacement.

$$
\left\{{ }_{s} x\right\}={ }_{s} \beta \cdot\left\{{ }_{s} u\right\} \cdot{ }_{s} \Delta
$$

${ }_{s} \Delta$ : The representative displacement for s-th mode.

Equations (11) and (12) are obtained with Equation (10).

$$
\begin{aligned}
& \sum m_{i} \cdot{ }_{s} u_{i}=\frac{1}{{ }_{s}^{\beta} \cdot{ }_{s} \Delta} \sum m_{i} \cdot{ }_{s} x_{i} \\
& \sum m_{i} \cdot{ }_{s} u_{i}{ }^{2}=\left(\frac{1}{{ }_{s} \beta \cdot{ }_{s} \Delta}\right)^{2} \sum m_{i} \cdot{ }_{s} x_{i}{ }^{2}
\end{aligned}
$$

Equation (13) is obtained from Equation (9) with using Equations (11) and (12).

$$
{ }_{s} \bar{M}=\frac{\left(\sum m_{i} \cdot{ }_{s} x_{i}\right)^{2}}{\sum m_{i} \cdot{ }_{s} x_{i}}
$$

The representative acceleration is derived as Equation (14) from Equation (8) when the external lateral force of ${ }_{s} P_{i}$ and relative displacement of ${ }_{s} x_{i}$ are obtained from a pushover analysis with the lateral force distribution shape defined as s-th mode shape.

$$
\left({ }_{s} \ddot{\Delta}+\ddot{X}_{0}\right)=\frac{{ }_{s} Q_{B}}{{ }_{s} \bar{M}}=\frac{\sum m_{i} \cdot{ }^{2} x_{i}{ }^{2}}{\left(\sum m_{i} \cdot{ } x_{i}\right)^{2}} \cdot \sum_{i=1}^{N}{ }_{s} P_{i}
$$

On the other hand, Equation (15) is derived from Equation (10).

$$
{ }_{s} \beta \cdot\left\{{ }_{s} u\right\}=\frac{1}{{ }_{s} \Delta}\left\{{ }_{s} x\right\}
$$
(15).

Equation (16) is derived from Equations (8), (13), and

$$
{ }_{s} \bar{M}=\frac{\sum_{i=1}^{N} m_{i} \cdot \frac{s^{x_{i}}}{{ }_{s} \Delta}\left({ }_{s} \ddot{\Delta}+\ddot{x}_{0}\right)}{{ }_{s} \ddot{\Delta}+\ddot{x}_{0}}=\frac{\left(\sum m_{i} \cdot{ }_{s} x_{i}\right)^{2}}{\sum m_{i} \cdot{ }_{s} x_{i}{ }^{2}}
$$

Therefore, the representative displacement ${ }_{s} \Delta$ is calculated as Equation (17) from Equation (16).

$$
{ }_{s} \Delta=\frac{\sum m_{i} \cdot{ }_{s} x_{i}^{2}}{\sum m_{i} \cdot{ }_{s} x_{i}}
$$

Since the calculated ${ }_{s} \ddot{\Delta}$ and ${ }_{s} \Delta$ are ${ }_{s} \ddot{q}_{0}$ and ${ }_{s} q_{0}$ in Equation (7), the relationship between ${ }_{s} \ddot{\Delta}$ and ${ }_{s} \Delta$ can be shown as Equation (18) with an s-th eigen angular frequency of ${ }_{s} \omega$.

$$
{ }_{s} \ddot{\Delta}+\ddot{x}_{0} \approx{ }_{s} \omega^{2} \cdot{ }_{s} \Delta
$$

When a building is in elastic, the maximum values of $\left|{ }_{s} \ddot{\Delta}+\ddot{x}_{0}\right|$ and $\left|{ }_{s} \Delta\right|$ are the same as the response spectra of $S_{a}$ and $S_{d}$ with an adequate damping coefficient. The curve of the relationship between $\left|{ }_{s} \ddot{\Delta}+\ddot{x}_{0}\right|$ and $\left|{ }_{s} \Delta\right|$ is defined as the capacity curve. The slope of the points on the capacity curve to the origin is square of the equivalent angular frequency, $\omega^{2}$, in the nonlinear range. The slope of the demand curve, which is the relationship between $S_{a}$ and $S_{d}$ is also square of the equivalent angular frequency. The intersection of the performance and demand curves has the same equivalent angular frequency, as shown in Equation (18).

\section{Capacity curve from the measured accelerations}

The calculation method for the capacity curve, which is the relationship between the representative acceleration, ${ }_{1} \ddot{\Delta}+\ddot{x}_{0}$, and the representative displacement, ${ }_{1} \Delta$, from the measured acceleration at each story is introduced. The displacement of each story is calculated from the measured accelerations with double integral, as shown in references 11) and 14). The Wavelet transformation is applied to take the predominant vibration as the first mode from the measured response.

\subsection{Outline of the Wavelet transformation ${ }^{12)}$}

The Wavelet transformation is one of the time-frequency analysis method, which shows the similarity of the signal $\mathrm{f}(\mathrm{x})$ and a mother wavelet mathematically. An original signal of $f_{0}$ is decomposed as $g_{1}$, which is a component with a particular frequency band, and residual of $f_{1}$ as shown in Equation (19).

$$
f_{0}=g_{1}+f_{1}
$$

By repeating this procedure, the original signal can be decomposed as Equation (20).

$$
f_{0}=g_{1}+g_{2}+g_{3}+\cdots+g_{n}+f_{n}
$$

Where the signal of $i-h$ decomposition, $g_{i}$, is called the signal for Rank i (i-th rank). The maximum rank of $n$ corresponds to the number of the signal, $\mathrm{N}$ as shown in Equation (21).

$$
\mathrm{n}=\log _{2} \mathrm{~N}
$$

Where $f_{n}$ is a deterministic value and the orthogonality of decomposed signals is proved mathematically.

The Wavelet transformation can be considered as a time-frequency analysis with the window of the mother wavelet. The width of the window in the temporal domain, $\Delta_{\hat{f}}$, and that in the frequency domain, $\Delta_{f}$, have the relation of uncertainty as shown in Equation (22).

$$
2 \Delta_{\hat{f}} \cdot 2 \Delta_{f} \geq 2
$$


When the time increment of an original signal, $f(x)$, is $\Delta_{\mathrm{t}}$, that of $g_{i}, \Delta_{t, i}$ is shown as Equation (23).

$$
\Delta_{t, i}=\Delta_{t} \times 2^{i}
$$

The Nyquist frequency of $\mathrm{i}$-th rank signal $g_{i}, \Delta_{f, i}$, is calculated as Equation (24).

$$
\Delta_{f, i}=\frac{1}{2 \Delta_{t} \cdot 2^{i}}
$$

The Wavelet transformation satisfies the minimum relation of uncertainty.

On the other hand, conventional filtering techniques such as band path filter ${ }^{12)}$ need to apply an appropriate base-line shift correction technique and to define filtering frequency in order to calculate displacement from measured acceleration. There is, however, no general method for them, so far. On the contrary, the Wavelet transformation can decompose any kind of signal in the temporal domain, and take any mode among the decomposed ranks based on its frequency.

\subsection{Capacity curve with the Wavelet transformation}

The objectives of applying the Wavelet transformation for a measured acceleration are to calculate reasonable response displacement with eliminating an error component contained in the measured acceleration and to take the predominant mode from the measured response ${ }^{11)}$, 14). The first mode can be taken from the measured response with the Wavelet transformation by taking the ranks of which frequency bands involve the predominant frequency of a building ${ }^{13)}$. The ranks taken as the first mode is called "predominant ranks" in this paper. The way to define the predominant ranks will be described in section 5.2.

The schematic of the measured acceleration and acceleration for each rank are shown in Fig. 10, where $\ddot{X}_{i}$ : absolute acceleration at i-th floor, $\ddot{x}_{i}$ : relative acceleration at i-th floor, $\ddot{x}_{0}$ : ground acceleration, ${ }_{1} \ddot{x}_{i}:$ relative acceleration for the first mode at ith-floor, and ${ }_{1} \ddot{x}_{0}$ : first mode component of the ground acceleration.
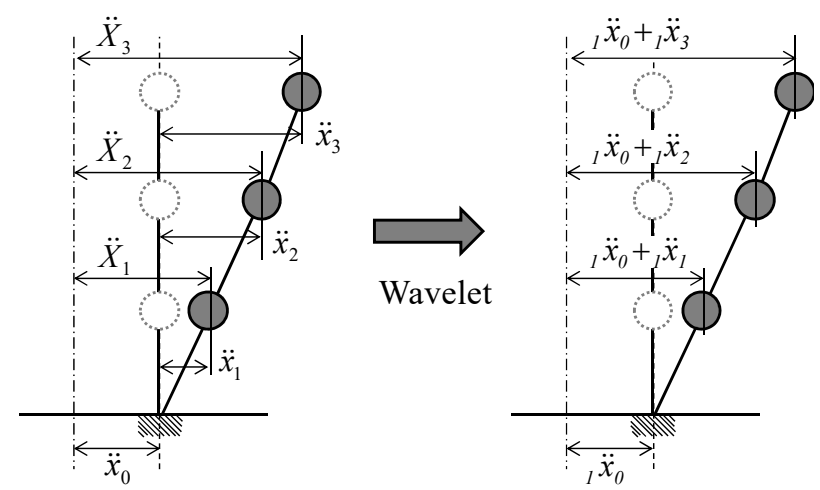

(a) Measured (b) First mode by Wavelet transformation Fig. 10 Schematic of the measured acceleration and acceleration for each rank with the Wavelet transformation
${ }_{1} \ddot{x}_{0}$ and ${ }_{1} \ddot{x}_{0}+{ }_{1} \ddot{x}_{i}$ can be calculated by applying the Wavelet transformation for the ground acceleration $\ddot{x}_{0}$ and measured absolute accelerations $\{\ddot{X}\}$.

The relative displacement to the basement is calculated with the double integral of the measured acceleration, as shown in Equation (25).

$$
{ }_{1} x_{i}=\iint\left({ }_{1} \ddot{x}_{0}+{ }_{1} \ddot{x}_{i}\right) d t^{2}-\iint{ }_{1} \ddot{x}_{0} d t^{2}
$$

Since the calculated relative displacement and acceleration with the Wavelet transformation do not contain higher mode component much, they are assumed to be the first mode responses.

\subsection{Capacity curve with measured acceleration}

It is already shown in section 5 that the representative acceleration ${ }_{s} \ddot{\Delta}+\ddot{x}_{0}$ and representative displacement ${ }_{s} \Delta$ can be derived as Equations (14) and (17) in the practical structural design, respectively. Since the proposed method in this paper is based on the first mode, Equations (26) and (27) are obtained from Equations (14) and (17), respectively.

$$
\begin{aligned}
& \left({ }_{1} \ddot{\Delta}+{ }_{1} \ddot{x}_{0}\right)=\frac{\sum m_{i \cdot 1} x_{i}{ }^{2}}{\left(\sum m_{i \cdot}{ }_{1} x_{i}\right)^{2}} \cdot \sum_{i=1}^{N}{ }_{1} P_{i} \\
& { }_{1} \Delta=\frac{\sum m_{i \cdot 1} x_{i}{ }^{2}}{\sum m_{i^{*}{ }_{1} x_{i}}}
\end{aligned}
$$

The representative displacement can be calculated as Equation (27) with using the relative displacement calculated as Equation (25).

The distribution shape of the external force, ${ }_{1} P$, in Equation (26) should be proportional to the first mode vector. As shown in Fig. 10 (b), the contribution of the ground acceleration to the story acceleration at each story is constant as ${ }_{1} \ddot{x}_{0}$. On the other hand, the distribution shape of the relative acceleration taken by the Wavelet transformation, ${ }_{1} \ddot{x}_{i}$, is proportional to the first mode vector. As a result, the distribution shape of the absolute acceleration, ${ }_{1} \ddot{x}_{0}+{ }_{1} \ddot{x}_{i}$, is not proportional to the first mode vector. In order to make the absolute acceleration proportional to the first mode vector, the stimulation factor of the first mode, ${ }_{1} \beta \cdot\left\{{ }_{1} u\right\}$, needs to be multiplied to the ground acceleration, ${ }_{1} \ddot{x}_{0}$. This means that the first mode of the unit vector of $\{1\}$ is multiplied to the ground acceleration. As a result, the external force proportional to the first mode vector is calculated as Equation (28).

$$
{ }_{1} P_{i}=m_{i}\left({ }_{1} \ddot{x}_{i}+{ }_{1} \beta \cdot{ }_{1} u_{i} \cdot{ }_{1} \ddot{x}_{0}\right)
$$

The representative acceleration is calculated as Equation (29) by substituting Equation 28 to Equation (26).

$$
\begin{gathered}
\left({ }_{1} \ddot{\Delta}+{ }_{1} \ddot{x}_{0}\right) \\
=\frac{\sum m_{i \cdot}{ }_{1} x_{i}{ }^{2}}{\left(\sum m_{i \cdot}{ }_{1} x_{i}\right)^{2}} \cdot \sum_{i=1}^{N}\left(m_{i} \cdot{ }_{1} \ddot{x}_{i}+m_{i} \cdot{ }_{1} \beta \cdot{ }_{1} u_{i} \cdot{ }_{1} \ddot{x}_{0}\right)
\end{gathered}
$$




$$
=\frac{\sum m_{i \cdot 1}{ }_{1}{ }^{2}}{\left(\sum m_{i} \cdot{ }_{1} x_{i}\right)^{2}} \sum_{i=1}^{N} m_{i} \cdot{ }_{1} \ddot{x}_{i}+{ }_{1} \ddot{x}_{0}
$$

As shown in Equation (29), only the relative acceleration term of the representative acceleration needs to be divided by the equivalent mass ratio when the representative acceleration is derived from the measured accelerations.

In Equations (27) and (28), the order of the mass $m_{i}$, is the same for denominator and numerator. Therefore, not the absolute value of mass but the ratio of the mass between floors is needed. If the usage of the building is unique, the floor area ratio may be used as the mass ratio.

Equations (27) and (29), however, do not give a practical capacity curve when the response displacement is very small. As an example, Fig. 11 shows the derived capacity curve with Equation (27) and (29) for a shaking table test results with the 3-story full-scale $\mathrm{R} / \mathrm{C}$ specimen $^{15)-19)}$. As shown in Fig. 11, the calculated representative acceleration became extremely large in small representative displacement range. The reason is that the distribution shape of the calculated relative displacement of each floor was not proportional to the first mode vector due to error contained in the measured values in the small displacement range, then the equivalent mass ratio became too small.

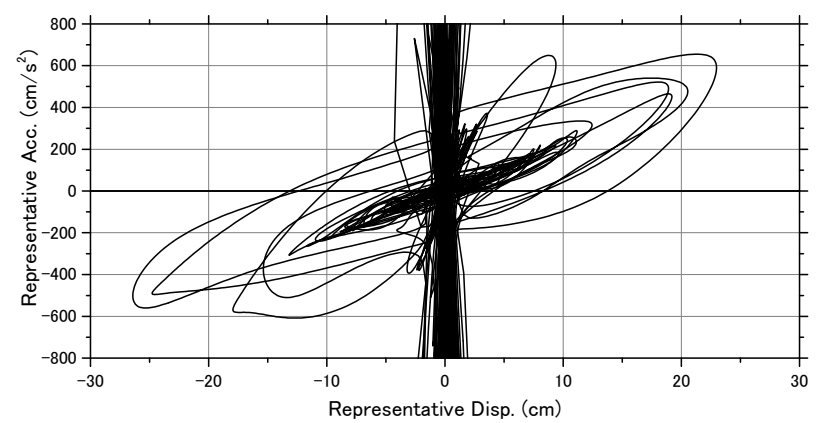

Fig. 11 Capacity curve derived from measured accelerations

The reason why the distribution shape was not proportional to the first mode vector was that small error contained in the measured acceleration that was not eliminated perfectly by the Wavelet transformation, which was amplified by the double integral. The error components exist all through the measured acceleration, but its effect is significant when the actual displacement is relatively small.

In order to avoid this effect of error, firstly "tentative" representative displacement, ${ }_{1} \Delta^{\prime}$, and "tentative" representative acceleration, ${ }_{1} \ddot{\Delta}^{\prime}+{ }_{1} \ddot{x}_{0}$, are calculated as Equations (30) and (31), respectively. The "tentative" capacity curve is defined as the relationship between tentative representative displacement and tentative representative acceleration.

$$
\begin{aligned}
{ }_{1} \Delta^{\prime}= & { }_{1} \Delta \cdot \frac{{ }_{1} \bar{M}}{M}=\frac{\sum m_{i \cdot 1}{ }_{1} x_{i}}{\sum m_{i}} \\
& \left({ }_{1} \ddot{\Delta}^{\prime}+{ }_{1} \ddot{x}_{0}\right)=\left({ }_{1} \ddot{\Delta} \cdot \frac{{ }_{1} \bar{M}}{M}+{ }_{1} \ddot{x}_{0}\right)
\end{aligned}
$$

These two equations are obtained by dividing the equivalent mass ratio (the ratio of the equivalent mass to the total mass) to Equation (27) and the first term of Equation (29). Since equivalent mass ratio is not used in Equations (30) and (31), no error such as shown in Fig. 11 does not happen in the small displacement range. The slope of the tentative capacity curve, however, does not coincide with the predominant frequency.

The procedure to calculate the capacity curve from the tentative capacity curve is introduced as follows;

(1) The tentative capacity curve is derived from the mass ratio and measured acceleration.

(2) A backbone curve is derived from the tentative capacity curve, where the backbone curve is the curve of the points that update maximum tentative representative displacement in the positive direction and minimum in the negative direction ${ }^{12}$.

(3) The equivalent mass ratio is calculated for the points of backbone curve derived in the procedure (2), then the capacity curve is calculated by dividing the tentative representative displacement and the first term of the tentative representative acceleration by the equivalent mass ratio.

\section{Demand curve with measured acceleration}

The demand curve, which is compared with the capacity curve to predict the maximum response, is calculated with using the ground acceleration, $\ddot{\mathrm{x}}_{0}$, measured at the basement of the building. The ground acceleration for the capacity curve, ${ }_{1} \ddot{x}_{0}$, is the first mode component of the ground acceleration taken by the Wavelet transformation. Therefore, the ground accelerations for the demand curve and capacity curve are not the same. Since ${ }_{1} \ddot{x}_{0}$ is taken from $\ddot{x}_{0}$ as the predominant component that affects the response of the building, the amounts of the demand for the predominant period of the building from both demand curves calculated with $\ddot{x}_{0}$ and ${ }_{1} \ddot{x}_{0}$ are almost the same.

Fig. 12 shows the calculated demand curves with the measured ground acceleration, $\ddot{x}_{0}$, during the shaking table test $\mathrm{t}^{(5)-19)}$, shown as "Original", and with the component of predominant ranks, ${ }_{1} \ddot{x}_{0}$, shown as "Rank6+7+8+9". The Nyquist frequencies of rank 5 to rank 8 are also superimposed to the figure. The figure shows that both demand curves coincide very well in the predominant frequency band of rank 5 to rank 8 . On the other hand, outside of the predominant frequency band, especially less than the period of $0.32 \mathrm{~s}$ (rank 5), the difference is significant. Since the period of less than 0.32 $\mathrm{s}$ is much shorter than the predominant period of the specimen, it is not effective to the evaluation of the maximum response. Therefore, the demand curve can be calculated with the measured acceleration at the basement, $\ddot{x}_{0}$. 


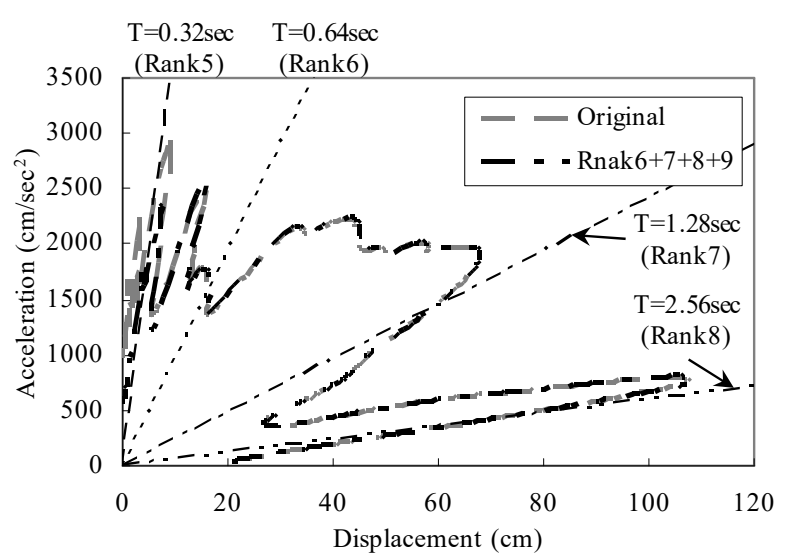

Fig. 12 Demand curves with $\ddot{x}_{0}$ and ${ }_{1} \ddot{x}_{0}$

There are K-Net seismograph stations close to the towers, roughly $5 \mathrm{~km}$ southeast of Hazawa Tower (KNG002) and roughly $2 \mathrm{~km}$ south of Higashi Oshima Tower (TKY013). Fig. 13 shows the calculated demand curves in the NS direction from the accelerations measured at the bottom of each tower and its nearest KNet station; the lines for the predominant frequencies are superimposed. In both cases, the demand curve for the acceleration measured at the bottom of the tower is smoother and smaller at the predominant frequency than that for the K-Net station, which was measured on the free field. From this, said it could be concluded that the effective earthquake force on a structure should not be defined according to the free-field record but rather according to the record from the base of the structure, which takes the soil-structure interaction into consideration.

\section{Performance curves derived from measured accelerations}

The calculated performance curves for Hazawa Tower and Higashi Oshima Tower during the Fukushima Ken Oki Earthquake the Ibaraki Ken North Earthquake are shown in Fig. 14 and Fig. 15, respectively. Fig. 14(a) shows clearly that Hazawa Tower remained elastic with a predominant frequency of $0.69373 \mathrm{~Hz}$ for the Fukushima Ken Oki Earthquake and $0.691064 \mathrm{~Hz}$ for the Ibaraki Ken North Earthquake, which are very close to the value from the transfer function shown in Fig. 5. Having three sensors in Hazawa Tower ensured good accuracy.

In contrast, the performance curve shown in Fig. 15 for Higashi Oshima Tower fluctuates. The slope of the straight-line fit corresponds to a frequency of roughly $0.385 \mathrm{~Hz}$ for the Fukushima Ken Oki Earthquake and $0.357 \mathrm{~Hz}$ for the Ibaraki Ken North Earthquake, which are less than the value of $0.415 \mathrm{~Hz}$ obtained from the transfer function shown in Fig. 7. A possible reason for the observed fluctuations is that too few sensors were installed in the tower. It is recommended to increase the number of sensors to improve the accuracy of the evaluation.

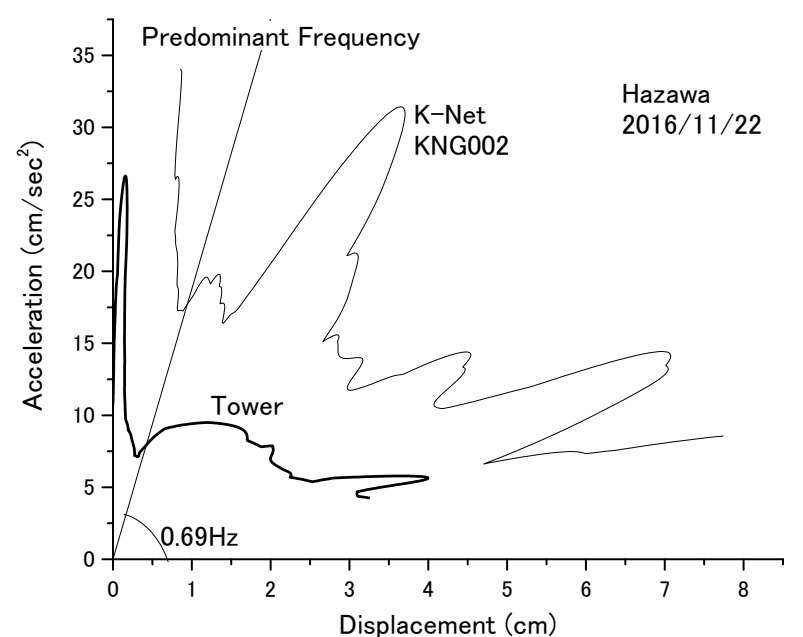

(a) Hazawa $(2016 / 11 / 22$, NS)

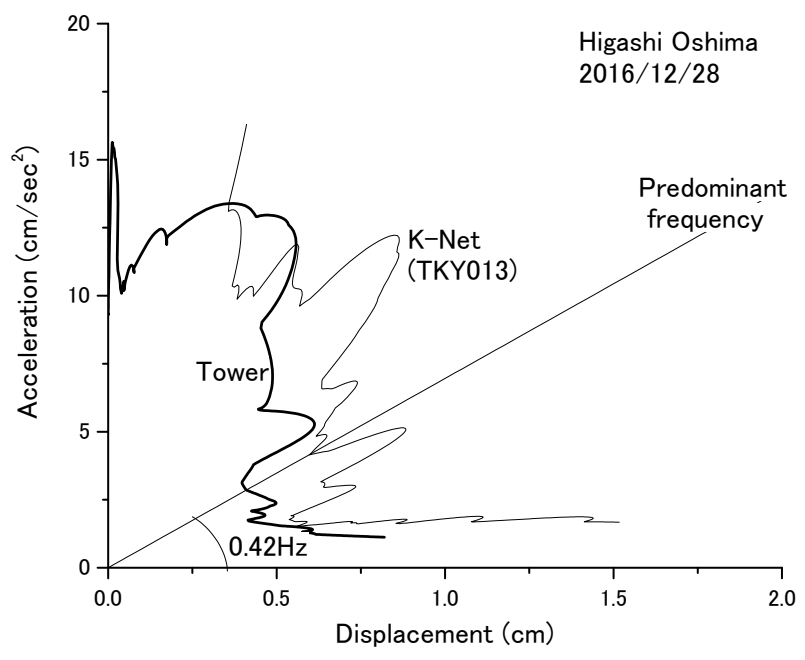

(b) Higashi Oshima (2016/12/28, NS)

Fig. 13 Demand curves (at tower base and at nearest K-Net station)

\section{Concluding remarks}

The dynamic response of two instrumented steel telecommunication towers that were in place during two major earthquakes in 2016 was investigated. The main results of the study are as follows.

1. The measurement systems worked successfully.

2. Because of a long-period component in each earthquake, both towers continued to oscillate even after the ground shaking had diminished or even ceased.

3. The demand curves associated with the base of each tower were smaller than those of the free field (KNet).

4. The performance curves of the towers were derived successfully from the measured accelerations, which showed that the towers remained elastic.

5. It is recommended that more accelerometers be placed in Higashi Oshima Tower. 


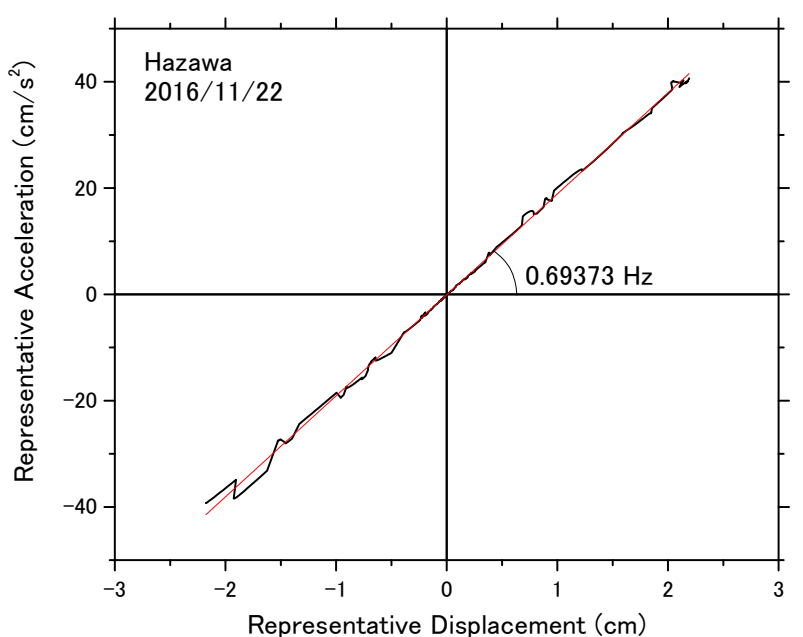

(a) Hazawa Tower, 2016/11/22, NS direction)

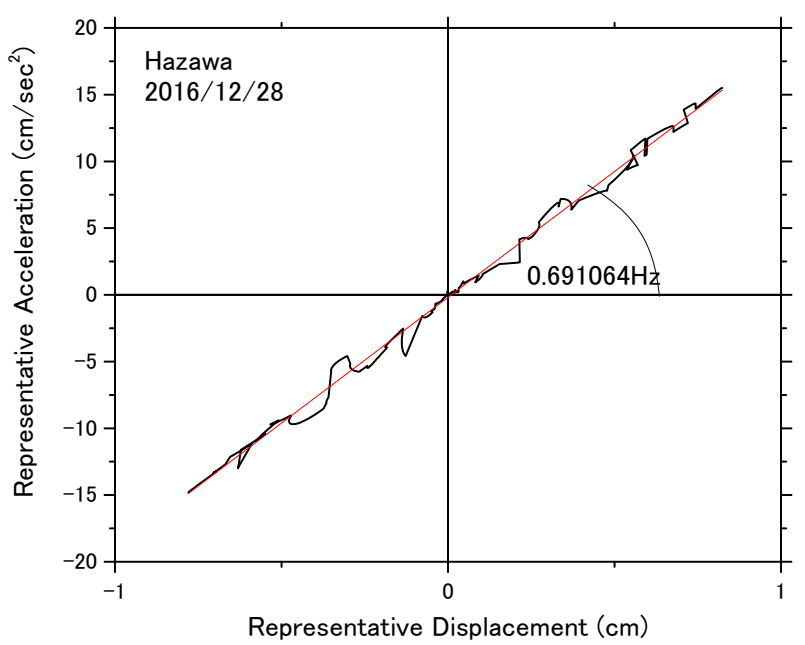

(b) Hazawa Tower, 2016/12/28, NS direction)

Fig. 14 Performance curves of Hazawa Tower

The author would like to thank the National Research Institute for Earth Science and Disaster Resilience for providing the data obtained by the Strong-motion Seismograph Networks (K-Net).

\section{References}

1. Kusunoki, K. and Teshigawara, M.: Development of Real-Time Residual Seismic Capacity Evaluation System - Integral Method and Shaking Table Test with Plain Steel Frame-, the 13th world conference on earthquake engineering, CD-Rom (2004).

2. Kusunoki, K., Elgamal, A., Teshigawara, M. and Conte, J. P.: Evaluation of structural condition using Wavelet transforms, the 14th World Conference on Earthquake Engineering, CD-Rom (2008).

3. Kusunoki, K., and Teshigawara, M.: A New Acceleration Integration Method to Develop A RealTime Residual Seismic Capacity Evaluation System, Journal of Structural And Construction Engineering, No.569, 119-126 (in Japanese) (2003):

4. Kusunoki, K., Tasai, A., Teshigawara, M.: Development of Building Monitoring System to Evaluate Residual Seismic Capacity after an

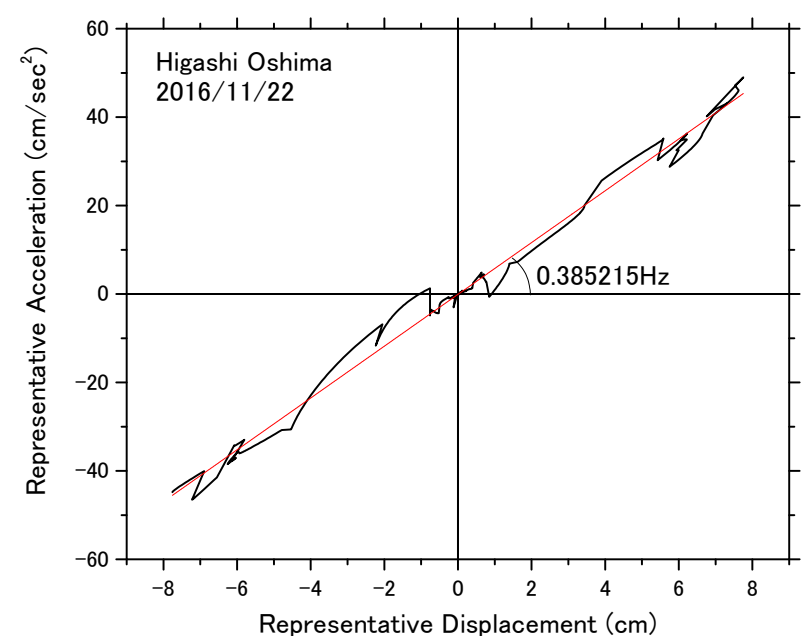

(a) Higashi Oshima Tower, 2016/11/22, NS direction

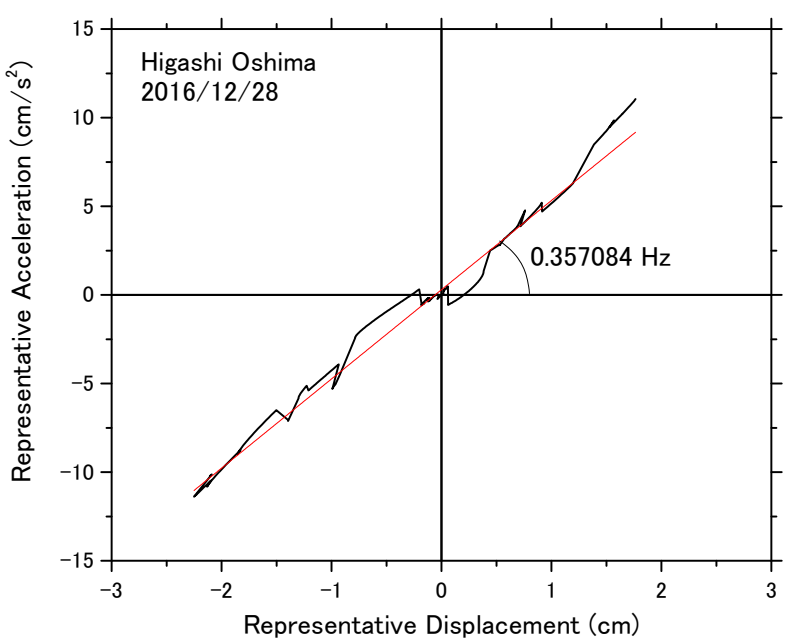

(b) Higashi Oshima Tower, 2016/12/28, NS direction

Fig. 15 Performance curves of Higashi Oshima Tower

Earthquake, the 15th World Conference on Earthquake Engineering, Digital (2012).

5. Kusunoki, K.: Damage Evaluation of A BaseIsolated Building With Measured Accelerations During Tohoku Earthquake, the 16th World Conference on Earthquake Engineering, Digital (2016).

6. Percival. D. B. and Walden, A. T.: Wavelet Methods for Time Series Analysis, Cambridge (2002).

7. Hiroshi KURAMOTO, Masaomi TESHIGAWARA, Norihide KOSHIIKA, and Hiroshi ISODA: Conversion of Multi-Story Building into Equivalent SDoF System and Its Predictability for Earthquake Response, Journal of Structural and Construct Engineering, AIJ, Vol. 546, pp.75-85, 2001.8

8. Building Guidance Division of Housing Bureau, Ministry of Land, Infrastructure, Transport, and Tourism: Calculation examples and commentaries for the capacity spectrum method ver. 2001, Kougaku Tosyo, 2001

9. Engineering affairs division of Ministry of Construction and Japan Association for Building Research Promotion: A guidelines for evaluating the 
seismic capacity of reinforced concrete buildings, Gihodo Press, 2000.07

10. Koichi Kusunoki, Daiki Hinata, Yuuki Hattori, and Akira Tasai: A new method for evaluating the realtime residual seismic capacity of existing structures using accelerometers: Structures with multiple degrees of freedom, Japan Architectural Review, Vol.1, No.1, pp.77-86, 2018.1

11. Manabu KAWAMURA, Koichi KUSUNOKI, Miho YAMASHITA, Yuki HATTORI, Daiki HINATA and Miguel Augusto DIAZ FIGUEROA : Study of A New Method to Compute The Performance Curve of Real Structures with Acceleration Sensors, in The Case of SDOF System Structures, Journal of Structural and Construct Engineering, AIJ, Vol. 688, pp1061-1069, 2013.06

12. Koichi KUSUNOKI and Masaomi TESHIGAWARA: A New Acceleration Integration Method to Develop A Real-Time Residual Seismic Capacity Evaluation System, Journal of Structural and Construct Engineering, AIJ, Vol. 569, pp.119126, 2003.7

13. Taiki HINATA, Koichi KUSUNOKI, Akira TASAI, and Manabu KAWAMURA: An Analytical Study on the Accuracy of the Capacity Curve Derived from the Measured Acceleration for Residual Seismic Capacity Evaluation System with Wavelet Transformation, Proceedings of the Japan Concrete Institute, Vol. 35, No. 2, pp.931-936, 2013

14. Haoran Pan \& Koichi Kusunoki, A wavelet transform - based capacity curve estimation approach using seismic response data, Structural Control and Health Monitoring, John Wiley and Sons Ltd., 2018
15. Masami ONOGUCHI, Shinichi MIYASHITA, Koichi KUSUNOKI, and Yoshiaki NAKANO: Shaking Table Test of Full-Scale Reinforced Concrete Three-Story Frame Structure (Part 1 Object and Outline of Specimen), Summaries of technical papers of Annual Meeting Architectural Institute of Japan. C-2, Structures IV, pp.707-708, 1995.8

16. Tomoyoshi IDE, Akihiko NAKAE, Shigeyuki AMITSU, and Fumitoshi KUMAZAWA: Shaking Table Test of Full-Scale Reinforced Concrete ThreeStory Frame Structure (Part 2 Outline of Experiment), Summaries of technical papers of Annual Meeting Architectural Institute of Japan. C2, Structures IV, pp.709-710, 1995.8

17. Isao KOGOMA, Manabu TOYOSHIMA, Toshihiro HAYASHIDA, and Chikahiro MINOWA : Shaking Table Test of Full-Scale Reinforced Concrete ThreeStory Frame Structure (Part 3 Results of Experiments), Summaries of technical papers of Annual Meeting Architectural Institute of Japan. C2, Structures IV, pp.711-712, 1995.8

18. Shigeyuki AMITSU, Keiji KITAJIMA, Hiroshi HOSOYA, and Hiroaki EDO: Shaking Table Test of Full-Scale Reinforced Concrete Three-Story Frame Structure (Part 4 Comparison between test and analytical results), Summaries of technical papers of Annual Meeting Architectural Institute of Japan. C2 Structures IV, pp.713-714, 1995.8

19. National Institute for Earth Science and Disaster Resilience : Experimental study on the collapse mechanism of reinforced concrete structure under a severe seismic shaking, 1993 annual report, 1993 